This item was submitted to Loughborough's Institutional Repository (https://dspace.lboro.ac.uk/) by the author and is made available under the following Creative Commons Licence conditions.

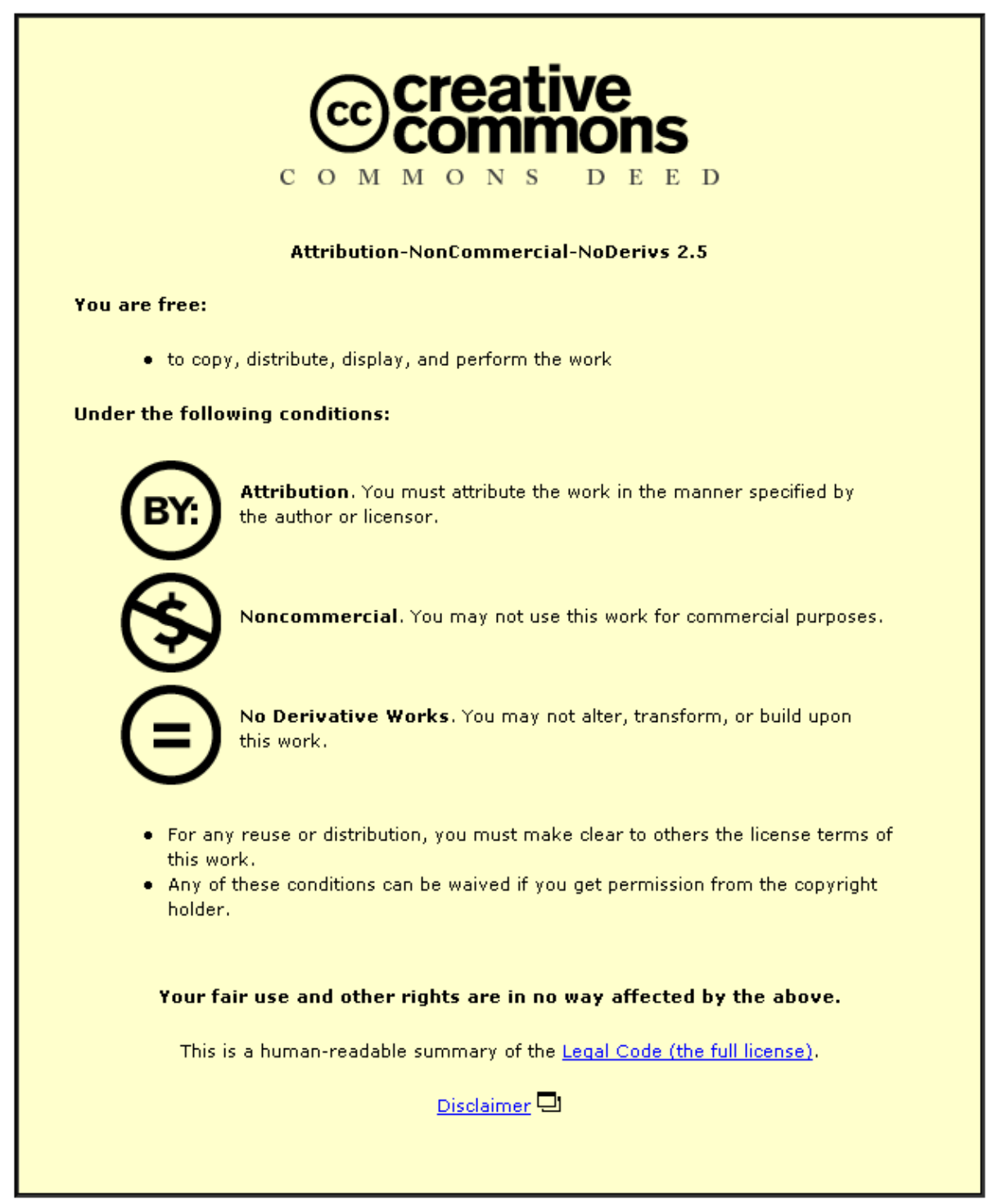

For the full text of this licence, please go to: http://creativecommons.org/licenses/by-nc-nd/2.5/ 


\title{
A COMBINED KALMAN FILTER AND CONSTANT MODULUS ALGORITHM BEAMFORMER FOR FAST-FADING CHANNELS
}

\author{
W. Pora, J.A. Chambers, and A.G. Constantinides \\ Dept. of Electrical \& Electronic Engineering, Imperial College, London SW7 2BT, UK. \\ E-mail:w.pora@ic.ac.uk
}

\begin{abstract}
Beamformers which use only the Constant Modulus Algorithm (CMA) are unable to track properly timevariant signals in fast-fading channels. The Kalman Filter (KF), however, has significant advantage in timevarying channels but needs a training sequence to operate. A combined CMA and KF algorithm is therefore proposed in order to utilise the advantages of both algorithms. The associated stepsize of the combination is also varied in accordance with the magnitude of the output. Simulations are presented to demonstrate the potential of this new approach.
\end{abstract}

\section{INTRODUCTION}

The Kalman filter (KF) [1] is the optimal filter for reconstructing signals travelling through linear timevarying channels. The $\mathrm{KF}$ has been employed for many functions and configurations. For example, in [2], the KF was used in decision feedback equalisers. The possibility of a combined KF and Least Mean Square (LMS) algorithm, which performs both equalisation and channel estimation was examined in [3]. This configuration achieves poor results, especially in fast-fading channels, because the tracking performance of the LMS algorithm is restrictive [4]. A combination of KF and Maximum-Likelihood Sequence Estimator (MLSE), in which the KF and MLSE were respectively used to track the channels and predict the signals, has been tested [5]. This is a more interesting approach because the KF tracks the channels potentially faster than LMS; and the MLSE, especially the multi-survivor algorithm, yields low Bit Error Rate (BER). However, its computational cost and block delay restrict its practical value. There has been an attempt in [6] to simplify the computation of the combination of $\mathrm{KF}$ and MLSE, this simplification still exhibits expensive computational cost, compared to those of adaptive Minimum Mean Squared Error (MMSE) type algorithms.

The CMA algorithm $[7,8]$ is one of the most powerful MMSE-type algorithms for stationary channels.
CMA needs neither training sequences, for learning the impulse response of the channels, nor array calibration, i.e., it can operate in a temporally and spatially blind mode. Furthermore, its computational complexity is very low at $O(N)$, where $N$ is the number of sensors. However, in fast-fading channels the amplitude of the received signals change very quickly. As for other gradient-descent type algorithms, the CMA algorithm has very limited tracking quality. Hence, it cannot track properly the outputs of the beamformer.

The next section states the problem of beamformers when the incoming sources suffer from fast-fading channels. In section 3 , a new beamformer using a combination of the $\mathrm{KF}$ and CMA algorithms is proposed. Simulations in section 4 will demonstrate the potential of the proposed algorithm. Finally, conclusions will be drawn.

\section{PROBLEM STATEMENT}

If $N$ sources, $s_{i}(k), i=1, \ldots, N$ impinge upon an array of $M$ sensors in directions which make angles $\theta_{i}(k)$ to a reference line of the array, the measurement signals, $\underline{\mathbf{x}}(k)=\left[x_{1}(k), \ldots, x_{M}(k)\right]^{\mathrm{T}}$, may be written as

$$
\underline{\mathbf{x}}(k)=\sum_{i=1}^{N} \underline{\mathbf{a}}\left(\theta_{i}(k)\right) G_{i}(k) s_{i}(k)+\underline{\mathbf{n}}(k)
$$

where $(\cdot)^{\mathbf{T}}$ denotes the transpose operator, $\underline{\mathbf{a}}(\theta), G_{i}(k)$ and $\underline{\mathbf{n}}(k)$ respectively represent a steering vector of the array, channel attenuation, and the additive measurement noise.

An output of a beamformer, $y(k)$, is a summation of a set of weighted measurement signals

$$
y(k)=\underline{\mathbf{w}}(k)^{\mathrm{H}} \underline{\mathbf{x}}(k)
$$

where $(\cdot)^{\mathrm{H}}$ denotes the Hermitian transpose operator, and $\underline{\mathbf{w}}(k)=\left[w_{1}(k), \ldots, w_{M}(k)\right]^{\mathrm{T}}$ represents the weight vector of the summation.

In this paper, all $s_{i}(k)$ are assumed to be zero-mean, white and have finite alphabets. The measurement 
noises are assumed to be zero-mean, white, Gaussian, and mutually unco:related with all measurement signals and each other.

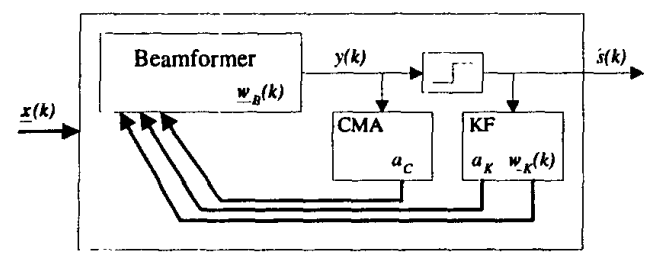

Figure 1: Structure of the beamformer using the combined $\mathrm{KF}$ and the CMA algorithms

\section{COMBINING CMA WITH THE KF}

An algorithm is required to control $\underline{\mathbf{w}}(k)$, or the beamformer, to capture some desired signal. If the channels are non-stationary, the algorithm has also to track the time variations. In fast-fading channels, $G_{i}(k)$, which has Rayleigh or Rician distribution, can change very quickly within a few milliseconds [9]. Therefore, the algorithm is required to have very good tracking quality.

\subsection{Kalman Filter}

A KF which minimises the Bayesian MSE cost

$$
J_{K F}=\mathrm{E}\left\{\|\underline{\mathbf{w}}(k)-\underline{\hat{\mathbf{w}}}(k)\|_{2}^{2} \mid d(k)\right\}
$$

and estimates $\underline{\mathbf{w}}(k)$ with $\underline{\hat{\mathbf{w}}}(k)$, given the transmitted sequence $d(k)$, is described by the following equations [10]

$$
\begin{aligned}
\underline{\mathbf{w}}(k) & =\mathbf{T}(k) \underline{\mathbf{w}}(k-1) \\
\mathbf{M}(k) & =\mathbf{T}(k) \mathbf{M}(k-1) \mathbf{T}(k)^{\mathrm{T}}+\mathbf{Q} \\
\underline{\mathbf{K}} & =\frac{\mathbf{M}(k) \underline{\mathbf{x}}(k)}{\sigma_{n}^{2}+\underline{\mathbf{x}}^{\mathrm{T}}(k) \mathbf{M}(k) \underline{\mathbf{x}}(k)} \\
\underline{\mathbf{w}}(k) & =\underline{\mathbf{w}}(k)+\left(d(k)-\underline{\mathbf{w}}(k)^{\mathrm{H}} \underline{\mathbf{x}}(k)\right)^{*} \underline{\mathbf{K}}^{*} \\
\mathbf{M}(k) & =\left(\mathbf{I}-\underline{\mathbf{K}} \underline{\mathbf{x}}(k)^{\mathrm{T}}\right) \mathbf{M}(k)
\end{aligned}
$$

where $\|\cdot\|_{2}$ and $(\cdot)^{*}$ respectively denote 2-norm and conjugate operators, $\mathbf{T}(k)$ and $\mathbf{Q}$ respectively represent a known $M \times M$ state transition matrix and a covariance matrix of the process noise, and $\mathbf{M}(k)$ is the prediction error matrix. The Kalman gain is expressed by $\underline{\mathbf{K}}$. The identity matrix and measurement noise power are respectively denoted by $I$ and $\sigma_{n}^{2}$.

Even though the KF has good tracking quality, it is normally a non-blind algorithm. Non-blind algorithms have very restricted applications in fast-fading channel environments because they need very frequent and long training sequences due to fast and large variations of the channels.

\subsection{Constant Modulus Algorithm}

The conventional CMA 2-2 algorithm [8] takes the following form

$$
\begin{aligned}
e(k) & =\left[\left|\underline{\mathbf{w}}(k)^{\mathbf{H}} \underline{\mathbf{x}}(k)\right|^{2}-R_{2}\right]\left[\underline{\mathbf{w}}(k)^{\mathrm{H}} \underline{\mathbf{x}}(k)\right] \\
\underline{\mathbf{w}}(k+1) & =\underline{\mathbf{w}}(k)-4 \mu e^{*}(k) \underline{\mathbf{x}}(k)
\end{aligned}
$$

where $R_{p}=\frac{\mathrm{E}\left\{|s(k)|^{2 p}\right\}}{\mathrm{E}\left\{|s(k)|^{p}\right\}^{2}}, p=2$, is a constant called the dispersion constant, and $\mu$ represents the stepsize of adaptation.

In order to track fast time-varying channels, the stepsize is required to be large. However, the stepsize cannot be too large due to the stability condition, especially in fast-fading channels which have substantial disturbance. Hence, the tracking capability of the CMA algorithm is limited.

\subsection{Variable Stepsizes}

The CMA algorithm tends to capture the source which has maximum power in the measurement signals [11]. In fast-fading channels, the power of one source may dominate at one time but not at another. Hence, the CMA algorithm may reconstruct one source at one time, and a different source at another time, thus, mixing up the sources would occur.

The blind KF can also switch the sources it reconstructs because the source which has maximum power also minimises, $\mathrm{E}\left\{\left\|\underline{\mathbf{w}}_{i}(k)-\hat{\mathbf{w}}_{i}(k)\right\|_{2}^{2} \mid \hat{s}_{i}(k)\right\}$. Since the $\mathrm{KF}$ is faster at tracking than CMA, it can potentially switch reconstructing the sources more often, in other words, its switching problem is more severe than that of CMA.

In [12], the stepsize of adaptation of CMA is timevarying and a function of the magnitude of the output of the beamformer.

$$
\mu_{v}(k)=\mu \mathbf{f}(|y(k)|)
$$

Function $\mathrm{f}(x)$ is designed to slow down the adaptation when the amplitude of the output of the beamformer is reduced. This usually occurs when the captured source fades. Therefore, this manoeuvre increases the tendency that a beamformer will capture only a single source.

\subsection{The New Combination}

The proposed combination of the KF and the CMA algorithms attempts to utilise advantages of both algorithm, e.g. blindness and tracking quality. The blind 
property is retained in this combination because the $\mathrm{KF}$ which tracks the weight vector, gets a training sequence from the output of the beamformer via a slicer as shown in Fig. 1. The KF then helps CMA to adapt effectively the weight vector of the beamformer.

A cost function of the combination is defined as

$$
\begin{aligned}
J^{\prime} & \triangleq \mathrm{E}\left\{\left(\left|\underline{\mathbf{w}}_{\mathbf{B}_{i}}^{\mathrm{H}}(k) \underline{\mathbf{x}}(k)\right|^{2}-R_{2}\right)^{2}\right\} \\
& +\kappa \mathrm{E}\left\{\left\|\underline{\mathbf{w}}_{\mathbf{B}_{i}}(k)-\underline{\mathbf{w}}_{\mathbf{B}_{i}}(k)\right\|_{2}^{2} \mid \hat{s}_{i}(k)\right\}
\end{aligned}
$$

where $\underline{\mathbf{w}}_{\mathbf{B}_{i}}(k)$ and $\underline{\mathbf{w}}_{\mathbf{B}_{i}}(k)$ represent respectively the weight vector of the beamformer and its estimate. The first term of the cost function is the cost function of the CMA and the other term is that of the KF with the scale of $\kappa$.

Then the adaptation of $\mathbf{w}_{\mathbf{B}_{i}}(k)$ with variable stepsize can be formulated as

$$
\begin{aligned}
y_{i}(k) & =\underline{\mathbf{w}}_{i}^{\mathbf{H}}(k) \underline{\mathbf{x}}(k) \\
\underline{\mathbf{w}}_{\mathbf{B}_{i}}(k+1) & =\underline{\mathbf{w}}_{\mathbf{B}}(k)+\mathrm{f}\left(\left|y_{i}(k)\right|\right)\left\{a_{C}+\kappa a_{K}\right\}(14)
\end{aligned}
$$

where $a_{C}=4 \mu\left(R_{2}-\left|y_{i}(k)\right|^{2}\right) y_{i}^{*}(k) \underline{\mathbf{x}}(k)$ and $a_{K}$ is the adaptation part obtained by the CMA and the KF algorithms.

The adaptation $a_{K}$ is the right term of $(7)$, but with $d(k)$ replaced with $\hat{s}_{i}(k)$, which is the sliced version of $y_{i}(k)$ by function $\mathrm{g}(\cdot)$. Equation (7) may be rewritten as

$$
\begin{aligned}
\hat{s}_{i}(k) & =\mathrm{g}\left(y_{i}(k)\right) \\
a_{K} & =\left(\hat{s}_{i}(k)-\underline{\mathbf{w}}_{\mathbf{K}}(k)^{\mathbf{H}} \underline{\mathbf{x}}(k)\right)^{*} \underline{\mathbf{K}}^{*} \\
\underline{\mathbf{w}}_{\mathbf{K}_{i}}(k) & =\underline{\mathbf{w}}_{i}(k)+\boldsymbol{a}_{K}
\end{aligned}
$$

where $\mathbf{w}_{\mathbf{K}_{i}}(k)$ is the weight vector of the KF in the combination (it also replaces $\underline{\mathbf{w}}(k)$ in (4)).

The value of $\kappa$ controls the amount of the blind KF part in the adaptation of the beamformer. A larger $\kappa$ improves adaptation speed, but if it is too large, it will cause the switching effect.

The weight $\mathbf{w}_{\mathbf{B}_{i}}(k)$ vector in the beamformer should be updated by $\mathbf{w}_{\mathbf{K}_{i}}(k)$ in the KF after some adaptations. If the update is too often, the switching effect will again occur.

\section{SIMULATIONS}

To demonstrate the tracking performance of the combination, an experiment is set. Consider that two uncorrelated signals travel through fast-fading channels to an array of two omnidirectional sensors in directions of $10^{\circ}$ and $20^{\circ}$ to the broad side of the array. The sources are modulated with Binary Phase Shift Keying (BPSK) and have the same average power $\sigma_{s}^{2}=1$ when they arrive at the array. They both suffer from

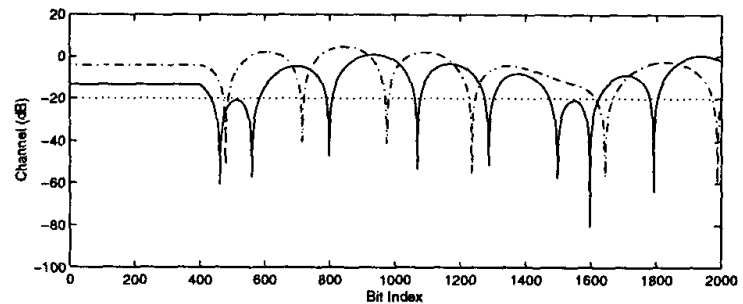

Figure 2: Fast-fading channels of source 1 (solid line) and source 2 (dot-dashed).

Doppler frequency spread of $60 \mathrm{~Hz}$, which corresponds to mobiles travelling about $45 \mathrm{mi} / \mathrm{hr}$, if the carrier frequency is $900 \mathrm{MHz}$. We assume that the baud rate of transmission is 24300 baud/s (the IS-54 standard). Simulation of the Rayleigh channels of both, is identical to the approach in [12], and is shown in Fig. 2. The additive noise at each sensor is zero-mean, white, Gaussian and uncorrelated with the sources and each other. Its power is calculated to be 0.01 of the average mean square power of the measurement signal at each sensor.

The real parts of the measurement signals are only passed to beamformers to avoid the mix-up effect [13, 14]. The tracking performance of bearnformers using only the CMA and that of beamformers using the combination will be compared. The weight vectors of all beamformers will be initialised to the correctly captured signals.

The stepsize, $\mu$ of CMA is chosen to be $0.01, \kappa$ is 0.2 . The weight vector $\mathbf{w}_{\mathbf{B}_{i}}(k)$ in the combination will be initialised by the weight vector of the KF $\underline{\mathbf{w}}_{i}(k)$ every 50 iterations. The function $\mathrm{f}(x)$ will return 1 if $x \geq 0.8,0.8$ if $0.5 \geq x<0.8,0.2$ if $0.2 \geq x<0.5$, and 0 otherwise.

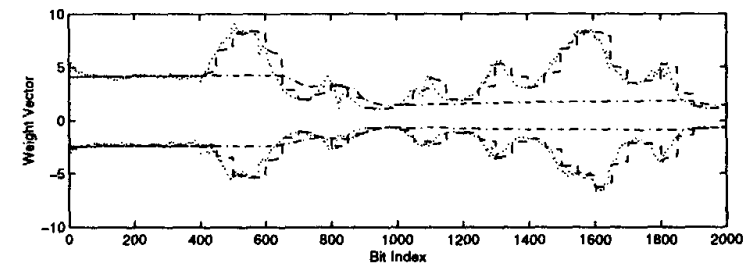

Figure 3: Weight vector (sensor 1 (upper lines) and sensor 2 (lower lines)), $\underline{\mathbf{w}}_{\mathbf{B}_{1}}(k)$ the proposed combined algorithm (dashed lines), $\mathbf{w}_{\mathbf{K}}(k)$ the Kalman filter (dotted lines), and that of the beamformer using only CMA (dot-dashed) 

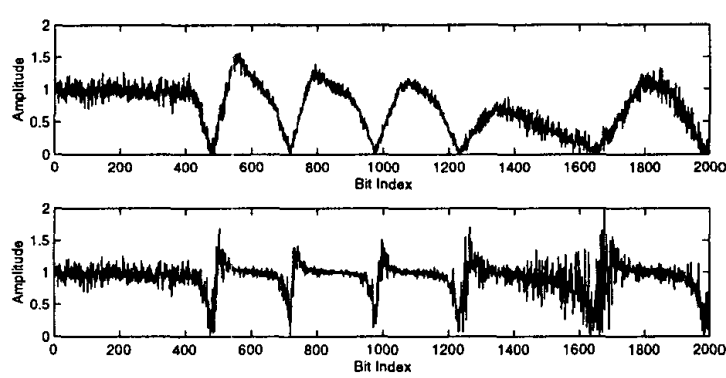

Figure 4: The magnitude of the outputs of the beamformer corresponding to source 2 using only CMA (upper graph) and using the proposed combination (lower graph)

Fig. 3 shows the movement of the weight vector $\mathbf{w}_{\mathbf{B}_{i}}(k)$ and $\mathbf{w}_{\mathbf{K}_{i}}(k)$ and that of the beamformer using only the CMA. It illustrates that CMA alone cannot follow the movement of the channels and the combination is potentially fast enough. The magnitude of an output of the beamformer using only CMA in Fig. 4, upper graph, shows that the CMA cannot retain the constant modulus property of the source. As a result of better tracking, the output of the beamformer using the combination in the lower graph exhibits much better constant modulus property.
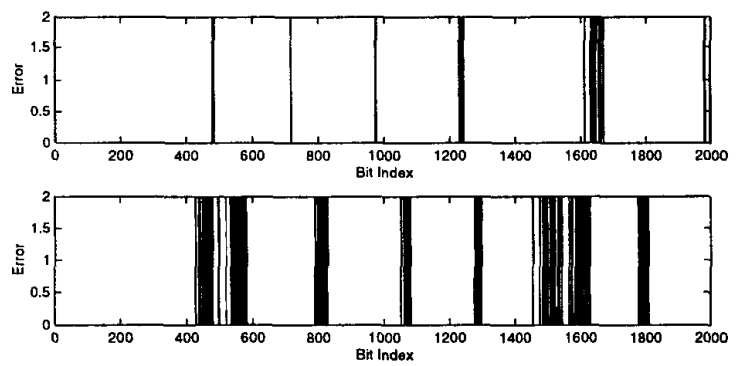

Figure 5: The symbol difference of the output of one beamformer and source 1 (upper graph), and that of the other beamformer and source 2 (lower graph)

The symbol difference of the outputs of beamformers and sources is shown in Fig. 5. The vertical lines in the graphs indicate bit errors. The places where the errors occur correspond to the deep fading of the sources below the measurement noise (see Fig. 2). These two graphs show that the combination with variable stepsize does not mix-up the sources.

\section{CONCLUSIONS}

A novel structure for a beamformer using a combination of the CMA and the KF algorithms has been proposed for tracking signals travelling through fast-fading channels. A variable stepsize has also been employed in the proposed algorithm. The simulations have supported the advantages of the proposed algorithm.

\section{REFERENCES}

[1] R. Kalman, "A new approach to linear filtering and prediction problems," Trans. ASME, Series D. J., Basic Eng., pp. 34-45, 1960.

[2] S. Chennakeshu, A. Narasimhan, and J. Anderson, "Decision feedback equalization for digital cellular radio," in IEEE International Conference on Communications, pp. 1492-1496, 1990.

[3] S. McLaughlin, "Adaptive equalisation via Kalman filtering," in IEE Procedings-F, pp. 388-396, 1991.

[4] S. Haykin, Adaptive Filter Theory. Prentice Hall, 3rd ed., 1991.

[5] G. D'Aria and V. Zingarelli, "Results on fast-Kalman and Viterbi adaptive equalizers for mobile radio with CEPT/GSM system characteristics," in Global Telecommunication Conference, pp. 815-819, 1988.

[6] M. Rollins and S. Simmons, "Simplified per-survivor Kalman processing in fast frequency-selective fading channels," IEEE Trans. Communications, pp. 544$553,1997$.

[7] D. Godard, "Self-recovering equalization and carrier tracking in two-dimensional data communication systems," IEEE Trans. on Communications, pp. 1867$1875,1980$.

[8] J. Treichler and B. Agee, "A new approach to multipath correction of constant modulus signals," IEEE Trans. Acoustic Speech and Signal Processing, pp. 459472, 1983.

[9] S. Redl, M. Weber, and M. Oliphant, An Introduction to GSM. Artech House Publishers, 1995.

[10] S. Kay, Fundamentals of Statistical Signal Processing Estimation Theory. Prentice Hall, 1993.

[11] J. Shynk and R. Gooch, "The constant modulus array for cochannel signal copy and direction finding," IEEE Trans. Signal Processing, pp. 652-660, 1996.

[12] A. Mathur, A. Keerthi, and J. Shynk, "A variable stepsize CM array algorithm for fast fading channel," IEEE Trans. Signal Processing, pp. 1083-1087, 1997.

[13] C. Papadias, "On the existence of undesirable global minima of godard equalizers," in ICASSP, pp. 39413944, 1997.

[14] W. Pora, S. Lambotharam, J. Chambers, and A. Constantinides, "A constant modulus array for real signals," To be appeared in EUSIPCO'98, 1998. 